
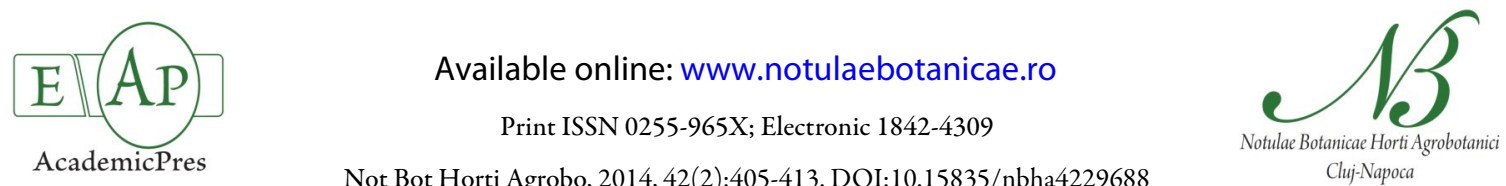

\title{
Alleviation of Salt Stress in Seedlings of Black Glutinous Rice by Seed Priming with Spermidine and Gibberellic Acid
}

\author{
Sumitahnun CHUNTHABUREE ${ }^{1}$, Jirawat SANITCHON², \\ Wattana PATTANAGUL ${ }^{1}$, Piyada THEERAKULPISUT ${ }^{1 *}$ \\ ${ }^{1}$ Department of Biology, Faculty of Science, Khon Kaen University, Khon Kaen 40002, Thailand; piythe@gmail.com (*correspondingauthor) \\ ${ }^{2}$ Department of Plant Science and Agricultural Resources, Faculty of Agriculture, Khon Kaen University, Khon Kaen 40002, Thailand
}

\begin{abstract}
This study was carried out to elucidate the spermidine $(\mathrm{Spd})$ and gibberellic acid $\left(\mathrm{GA}_{3}\right)$ priming-induced physiological and biochemical changes responsible for induction of salinity tolerance in two rice (Oryza sativa L.) cultivars, namely 'Niewdam Gs. no. 00621' (salt tolerant) and 'KKU-LLR-039' (salt sensitive). The seeds of the two cultivars were primed separately with distilled water, $1 \mathrm{mM}$ Spd or $0.43 \mathrm{mM} \mathrm{GA}_{3}$. Primed seeds were germinated and the resultant seedlings were hydroponically grown for 14 days before being exposed to salinity stress $(150 \mathrm{mM} \mathrm{NaCl})$ for 10 days. Seed priming with Spd or $\mathrm{GA}_{3}$ slightly improved salt-induced reductions in growth, anthocyanin and chlorophyll contents of the seedlings. Salt stress induced pronounced increases in $\mathrm{Na}^{+} / \mathrm{K}^{+}$ratio, proline and $\mathrm{H}_{2} \mathrm{O}_{2}$ contents, particularly in the sensitive cultivar. The levels of these saltsensitivity physiological indicators tended to be mitigated by priming with $\mathrm{Spd}$ and $\mathrm{GA}_{3}$. Salt-stressed seedlings grown from seeds primed with these growth regulators also possessed higher phenolic contents and greater antioxidant capacity than the control seedlings. Based on all growth and physiological data, Spd tended to be more effective than $\mathrm{GA}_{3}$ in improving salt tolerance in both rice cultivars.
\end{abstract}

Keywords: antioxidant capacity, $\mathrm{Na}^{+} / \mathrm{K}^{+}$, polyamine, seed pretreatment

\section{Introduction}

Soil salinity is a major environmental stress that drastically affects plants growth and productivity of several crops by creating low osmotic potential outside the seeds, thereby inhibiting or slowing down seed germination and later retarding seedling growth because of both osmotic and ion toxicity effects (Khajeh-Hosseini et al., 2003). Numerous attempts have been made to improve the salinity tolerance of a variety of crops by traditional breeding programs (Duan et al., 2008), but the progress to develop such salt-resistant plants is very slow and commercial success is limited. Therefore, the use of some other simple, costeffective methods, such as seed priming could be an attractive approach to overcome growth retardation of young plants in the salt-affected soils. Seed quality, seed germination rate and seedling vigor are important factors for sustainable crop production particularly under adverse environmental conditions (Koornneef et al., 2002; Sun et al., 2007). Priming is a process by which seeds are exposed to restricted water availability under controlled conditions, which allows some pre-germination metabolic activities (physiological and chemical) to proceed, before germination is completed, followed by re-drying for short-term storage before sowing (Bradford, 1986; Farooq et al., 2010). The effectiveness of different priming agents, however, varies under different stresses as well as in different crop species. Plant hormones are active members of the signal cascade involved in the induction of plant stress responses (Pedranzani et al., 2003). Several chemicals have been used for seed priming (Afzal et al., 2012). Of various priming agents employed for seed priming, plant growth regulators have gained much attention from researchers all over the world because of their consistent effects on seed germination as well as growth of a variety of plant species (Farooq et al., 2007; Iqbal and Ashraf, 2013).

Polyamines (PAs), including the triamine spermidine $\left(\mathrm{Spd}^{3+}\right)$, tetramine spermine $\left(\mathrm{Spm}^{4+}\right)$ and their obligate precursor, the diamine putrescine $\left(\mathrm{Put}^{2+}\right)$ are now regarded as plant growth regulators and secondary messenger in signaling pathways (Kusano et al., 2008). Because of their cationic nature at physiological $\mathrm{pH}, \mathrm{PAs}$ are able to interact with proteins, nucleic acids, membrane phospholipids and cell wall constituents, thereby stabilizing these molecules (Bouchereau et al., 1999). Apart from their implication in growth and development, PAs have been reported to be involved in defense response to biotic and abiotic stresses (Alcázar et al., 2010). Exogenously applied PAs have been reported to substantially enhance salt tolerance in rice plants (Chattopadhyay et al., 2002). Moreover, Khan et al. (2012) 
reported that seed priming with Put, Spd and Spm improved seed germination as well as promoted early seedling growth in hot pepper.

Gibberellins (GAs) play an important role in many essential plant growth and development processes, including seed germination, stem elongation, leaf expansion, flower and fruit development, and floral transition (Razem et al., 2006). The mechanisms by which Spd and $\mathrm{GA}_{3}$ priming could induce salt tolerance in plants have been widely proposed (Khan et al., 2012; Iqbal and Ashraf, 2013). However, studies concerning Spd and $\mathrm{GA}_{3}$ priminginduced physiological and biochemical changes in rice in saline conditions are scarce and little is known about the effect of GAs on physiological and biochemical changes in induction of salt tolerance in rice plants. Thus, investigating the possibility of exogenously applied Spd and $\mathrm{GA}_{3}$ in induction of salt tolerance in rice is of great importance. The overall objective of this study was to elucidate the Spd and $\mathrm{GA}_{3}$ priming-induced physiological and biochemical changes responsible for induction of salinity tolerance in two rice (Oryza sativa L.) cultivars, namely, 'Niewdam Gs. no. 00621' and 'KKU-LLR-039'.

\section{Materials and methods}

\section{Plant materials and priming treatments}

Two black, glutinous rice (O. sativa L.) cultivars, 'Niewdam Gs. no. 00621' (salt-tolerant) and 'KKU-LLR-039' (saltsensitive) were used in the present study. The seeds were obtained from the Department of Plant Science and Agricultural Resources, Faculty of Agriculture, Khon Kaen University, Thailand. Solutions of $1 \mathrm{mM}$ Spd and $0.43 \mathrm{mM}$ (150 $\left.\mathrm{mg} \mathrm{L}^{-1}\right) \mathrm{GA}_{3}$ were utilized for seed priming. Distilled water was used for hydro-priming. Prior to priming treatments, rice seeds were surface-sterilized in $0.5 \%$ sodium hypochlorite for $10 \mathrm{~min}$ and rinsed thrice with distilled water. The rice seeds were primed separately, in the dark, in $5 \mathrm{ml}$ solutions of $\mathrm{GA}_{3}$, Spd and distilled water for 24 hours at ambient temperature. Following pre-soaking treatments, the seeds were rinsed with distilled water and air-dried for 48 hours at ambient temperature to their original moisture content (about 9-10\%). The primed and non-primed seeds were sown for 3 days to produce seedlings. The obtained seedlings were transferred to continuously aerated Yoshida's solution in a greenhouse with twenty seedlings per treatment. The solution $\mathrm{pH}$ was adjusted to 5.0 and was renewed at 4-days intervals. Following a 14-day planting period, the seedlings were transferred to Yoshida's solution supplemented with $150 \mathrm{mM} \mathrm{NaCl}$ for 10 days. The seedlings that were cultivated in the absence of additional $\mathrm{NaCl}$ were set as controls. Seedlings were harvested after 10 days, separated into aerial and radical parts for growth analyses, including shoot length (SL), root length (RL), fresh weight (FW) and dry weight (DW) of shoot and root. To measure the seedling DW, the samples were dried in a hot-air oven at $70^{\circ} \mathrm{C}$ for 3 days. Leaf samples were collected and stored at $-80^{\circ} \mathrm{C}$ for further use in physiological and biochemical analyses.

\section{Measurement of ion concentration}

Following 3 days of oven-drying of seedling shoots at $60^{\circ} \mathrm{C}$, about $0.5 \mathrm{~g}$ of each dried powdered sample was successively digested with $10 \mathrm{ml}$ of nitric acid at $300^{\circ} \mathrm{C}, 5 \mathrm{ml}$ of perchloric acid at $200{ }^{\circ} \mathrm{C}$ and $20 \mathrm{ml}$ of $6 \mathrm{M}$ hydrochloric acid at $200^{\circ} \mathrm{C}$, for $30 \mathrm{~min}$. The concentrations of $\mathrm{Na}^{+}$and $\mathrm{K}^{+}$were analyzed using atomic absorption spectroscopy.

\section{Analysis of pigment accumulation}

Chlorophyll content was determined following the method described by Arnon (1949) with minor modifications. About $0.5 \mathrm{~g}$ of leaf samples were extracted for chlorophyll by soaking the leaf samples in $10 \mathrm{ml}$ of $80 \%$ acetone overnight in the dark. The extract was centrifuged at $10000 \times \mathrm{g}$ for $5 \mathrm{~min}$ and the absorbance of the supernatant was read at 645 and 663 $\mathrm{nm}$ using a spectrophotometer and chlorophyll concentrations were then calculated and expressed as $\mathrm{mg} \mathrm{g}^{-1} \mathrm{FW}$, using the following equations:

$$
\begin{aligned}
& \text { Total-Chl }=\left[20.2\left(\mathrm{~A}_{645}\right)+8.02\left(\mathrm{~A}_{663}\right)\right] \times \mathrm{x}(\mathrm{V} / 1000 \mathrm{~W}), \\
& \text { Chl- } a=\left[12.7\left(\mathrm{~A}_{663}\right)-2.69\left(\mathrm{~A}_{645}\right)\right] \times(\mathrm{V} / 1000 \mathrm{~W}), \\
& \text { Chl- } b=\left[22.9\left(\mathrm{~A}_{645}\right)-4.69\left(\mathrm{~A}_{663}\right)\right] \times(\mathrm{V} / 1000 \mathrm{~W}) .
\end{aligned}
$$

where $\mathrm{A}_{645}$ and $\mathrm{A}_{663}$ represent absorbance of chlorophyll concentrations extract at 645 and $663 \mathrm{~nm}$ respectively, $\mathrm{V}$ is the total extract volume and $\mathrm{W}$ is the leaf fresh weight.

Total anthocyanin content (TAC) was estimated according to the method described by Abdel-Aal and Hucl (1999). Approximately $0.1 \mathrm{~g}$ of leaf tissues were soaked for 72 hour in $10 \mathrm{ml}$ of acidified ethanol (ethanol : $1 \mathrm{~N} \mathrm{HCl}, 85: 15$ $\mathrm{v} / \mathrm{v})$. The suspension was filtered through Whatman No.1 filter paper and absorbance was measured at $535 \mathrm{~nm}$.

\section{Analysis of proline content}

Proline content was quantified according to the method described by Bates et al. (1973) with minor modifications. About $0.1 \mathrm{~g}$ fresh weight of leaf tissues were homogenized and extracted utilizing $5 \mathrm{ml}$ of $3 \%$ aqueous sulfosalicylic acid. Then $2 \mathrm{ml}$ of extract were reacted with $2 \mathrm{ml}$ of acid ninhydrin and 2 $\mathrm{ml}$ of glacial acetic acid and boiled at $100{ }^{\circ} \mathrm{C}$ for 1 hour. The reaction was quenched by placing the tubes rapidly on ice. The resulting solutions were extracted with toluene, and the absorbance of the toluene fraction was monitored at $520 \mathrm{~nm}$. Proline content was estimated with reference to a calibration curve and expressed as $\mu \mathrm{gg}^{-1}$ tissue FW.

\section{Analysis of $\mathrm{H}_{2} \mathrm{O}_{2}$}

For determination of $\mathrm{H}_{2} \mathrm{O}_{2}$ concentration, $0.1 \mathrm{~g}$ fresh weight of leaf tissues were extracted with $3 \mathrm{ml}$ of $0.1 \%$, w/v trichloroacetic acid (TCA) in an ice bath and centrifuged at $12,000 \times \mathrm{g}$ for $15 \mathrm{~min}$ and $0.5 \mathrm{ml}$ of the supernatant was added to $0.5 \mathrm{ml}$ of $10 \mathrm{mM}$ potassium phosphate buffer $(\mathrm{pH} 7.0)$ and $1 \mathrm{ml}$ of $1 \mathrm{M}$ potassium iodide. The absorbance of supernatant was read at $390 \mathrm{~nm}$. The content of $\mathrm{H}_{2} \mathrm{O}_{2}$ was estimated based on a standard curve (Velikova et al., 2000).

\section{Extraction procedure to determine the antioxidant properties}

The method of extraction for determination of antioxidant properties of rice leaf was adapted from Sultana et al. (2009). Rice leaf samples $(0.1 \mathrm{~g})$ were extracted with $10 \mathrm{ml}$ of $80 \%$ aqueous ethanol for 48 hours at room temperature. After centrifugation at $10,000 \times \mathrm{g}$ for $10 \mathrm{~min}$, the suspension was filtered through Whatman No.1 filter paper and used to determine total phenolic content (TPC) and antioxidant capacity and the measurements were replicated four times. 
407

Analysis of TPC

Total phenolic content was assayed by Folin-Ciocalteu's reagent method (Singleton et al., 1999). Briefly, $120 \mu \mathrm{l}$ of leaf extract was placed into test tubes and then $600 \mu \mathrm{l}$ of freshly diluted 10-fold Folin-Ciocalteu's reagent was added. After $2 \mathrm{~min}, 480 \mu \mathrm{l}$ of sodium carbonate solution $\left(75 \mathrm{~g} \mathrm{~L}^{-1}\right)$ was added. The mixtures were vigorously shaken and allowed to stand for $40 \mathrm{~min}$. The absorbance of the resulting blue color was measured at $760 \mathrm{~nm}$. Gallic acid was used as standard and TPC and was expressed as mg gallic acid (GAE) equivalent per $1 \mathrm{~g}$ leaf FW ( $\left.\mathrm{mg} \mathrm{GAE} \mathrm{g}^{-1}\right)$.

\section{2,2' -diphenyl-1-picrylhydrazyl (DPPH) radical-scavenging} assay

The DPPH radical-scavenging ability of rice extracts was estimated according to the method described by BrandWilliams et al. (1995). The reaction mixture contained $1.5 \mathrm{ml}$ DPPH working solution (4.73 mg of DPPH in $100 \mathrm{ml}$ ethanol HPLC-grade) and $100 \mu \mathrm{l}$ rice leaf extract. The mixture was shaken and incubated for 1 hour in the dark at room temperature. The absorbance was read at $515 \mathrm{~nm}$ relative to the control using a spectrophotometer. The percentage of radical scavenging was calculated according to the following equation:

\% DPPH scavenging activity $(\%)=\left[\left(\mathrm{Abs}_{\text {connol }}-\mathrm{Abs}_{\text {smplc }}\right)\right] /$ $\left(\right.$ Abs $\left.\left._{\text {connol }}\right)\right] \times 100$,

where $\mathrm{Abs}$ contol is the absorbance of DPPH + ethanol;

Abs sample is the absorbance of DPPH radicals + sample extract.

2,2'-azinobis (3-ethylbenzotbiazoline 6-sulfonic acid) $\left(A B T S^{+}\right)$scavenging assay

Radical cation $\mathrm{ABTS}^{+}$scavenging assay was performed following the method described by Re et al. (1999) with minor modifications. A stable stock solution of ABTS radicals was prepared by reacting $7 \mathrm{mM}$ aqueous solution of ABTS with potassium persulfate in the dark at room temperature for 12-16 hours before use. Rice leaf extract $(120 \mu \mathrm{l})$ was allowed to react with $1.5 \mathrm{ml}$ of a diluted ABTS radical solution (absorbance of $0.70 \pm 0.02$ at $734 \mathrm{~nm}$ ). The absorbance at $734 \mathrm{~nm}$ of the mixture was measured after a $30-\mathrm{min}$ reaction period. The percentage of radical scavenging was calculated according to the below-given equation:

$\%$ ABTS scavenging activity $=\left[\left(\mathrm{Abs}_{\text {connol }}-\mathrm{Abs}_{\text {sumple }}\right) /(\mathrm{Abs}\right.$ conrol) $] \times 100$,

where Abs control is the absorbance of $\mathrm{ABTS}^{+}$radical + ethanol;

Abs sample is the absorbance of $\mathrm{ABTS}^{+}$radical + sample extract.

\section{Ferric reducing antioxidant power (FRAP) assay}

The method adapted from Benzie and Strain (1996) was used for the FRAP assay. A $200 \mu \mathrm{l}$ volume of rice leaf extract was mixed with $1.3 \mathrm{ml}$ of FRAP reagent and the mixture was incubated at $37^{\circ} \mathrm{C}$ for $30 \mathrm{~min}$. Following incubation, the absorbance of the mixture was read at $595 \mathrm{~nm}$. The FRAP reagent was freshly prepared as required and consisted of $0.3 \mathrm{M}$ acetate buffer ( $\mathrm{pH}$ 3.6), $10 \mathrm{mM} \mathrm{2,} \mathrm{4,} \mathrm{6-tripyridyl-s-triazine}$ (TPTZ) in $40 \mathrm{mM} \mathrm{HCl}$ and $20 \mathrm{mM} \mathrm{FeCl}_{3}(10: 1: 1$, in $\mathrm{v} / \mathrm{v} / \mathrm{v})$. The antioxidant activity was expressed as mmol of Trolox equivalents (TE) per $100 \mathrm{~g} \mathrm{FW}$.

\section{Statistical analysis}

The experiments were arranged as a factorial experiment based on a completely randomized design with 4 replications. Treatments included two rice cultivars, four priming treatments (unprimed, hydropriming, priming with Spd and with $\left.\mathrm{GA}_{3}\right)$ and two levels of salinity $(0$ and $150 \mathrm{mM} \mathrm{NaCl})$. The data was subjected to an analysis of one-way and two-way ANOVA and all means were separated at the $p<0.05$ level using the Duncan's multiple range test. All calculations and data analyses were performed using the SPSS 16.0 for Windows software package. Pearson's correlation was calculated for the relationship between seedling growth, physiological parameters and also antioxidant capacity of seedlings grown from primed and non-primed seeds under saline conditions.

\section{Results and discussions}

\section{Spd and $\mathrm{GA}_{3}$ priming modulates growth parameters}

The imposition of salt stress caused a significant reduction in seedling growth of both rice cultivars (Fig. 1). A significant increase in shoot length was observed in 'KKU-LLR-039' when Spd was used as a priming agent. Analysis of variance (Tab. 3) showed that all six growth traits were significantly affected by salt stress while only SL, SFW and SDW were significantly affected by different priming treatments and there were no cultivars and priming treatments $(\mathrm{C} \times \mathrm{P})$ interaction for all of the growth traits. Priming with Spd and $\mathrm{GA}_{3}$ improved root length of both cultivars although not statistically significantly. Gibberellins are generally involved in growth and development; they control seed germination, leaf expansion, stem elongation and flowering (Magome et al., 2004). Gibberellic acid has been reported to be helpful in enhancing growth under saline conditions in rice (Wen et al., 2010), corn (Ghodrat and Rousta, 2012), wheat (Iqbal and Ashraf, 2013) and cucumber (Radhakrishnan and Lee, 2014). Of the two priming agents applied, Spd was more effective in enhancing salt tolerance in both cultivars. Furthermore, exogenous Spd also has been reported to be effective in enhancing rice growth under saline conditions (Saleethong et al., 2011; Roychoudhury et al., 2011).

\section{Spd and $\mathrm{GA}_{3}$ priming alters $\mathrm{Na}^{+} / \mathrm{K}^{+}$ratios}

Salt stress caused a significant increase in $\mathrm{Na}^{+} / \mathrm{K}^{+}$ratios in seedlings of both cultivars grown from unprimed seeds; by $146.65 \%$ for the salt-tolerant cultivar 'Niewdam Gs. no. 00621 ' and by $188.98 \%$ for the salt-sensitive cultivar 'KKU-LLR-039' (Tab. 1). Priming with Spd and GA $\mathrm{A}_{3}$ significantly reduced the seedling $\mathrm{Na}^{+} / \mathrm{K}^{+}$ratios in both cultivars. However, the interaction between cultivars and priming treatments $(\mathrm{C} \times \mathrm{P})$ was not significant (Tab. 3). Considering consistency and effectiveness of the priming treatments, Spd treatments were more effective in reducing the seedling $\mathrm{Na}^{+} / \mathrm{K}^{+}$ratios in both cultivars in saline conditions. Rice plants with salt-resistant characteristics commonly possess superior ion homeostasis strategies, particularly a high $\mathrm{K}^{+} / \mathrm{Na}^{+}$ratios, through exclusion, compartmentation and partitioning of $\mathrm{Na}^{+}$in shoots or roots (Blumwald, 2000). Under saline condition, the $\mathrm{Na}^{+} / \mathrm{K}^{+}$ratios were positively correlated with proline and $\mathrm{H}_{2} \mathrm{O}_{2}$ but negatively correlated with SL, Chl- $b$, TAC, TPC and antioxidant capacities (Tab. 4). Based on our study, pre-treatment of seeds 
with Spd and $\mathrm{GA}_{3}$ was noted to alleviate the inhibitory effect of salt stress in both cultivars under saline conditions. The findings in our study were well-supported by an earlier study (Iqbal and Ashraf, 2013) that seed priming with $\mathrm{GA}_{3}$ decreased $\mathrm{Na}^{+}$concentrations in the roots and shoots of two wheat cultivars, namely MH-97 (salt-intolerant) and Inqlab-91 (salttolerant), when grown under saline conditions. Zhu et al. (2006) also reported that the exogenous application of PAs, specifically Spd inhibits $\mathrm{Na}^{+}$transport in barley seedlings under

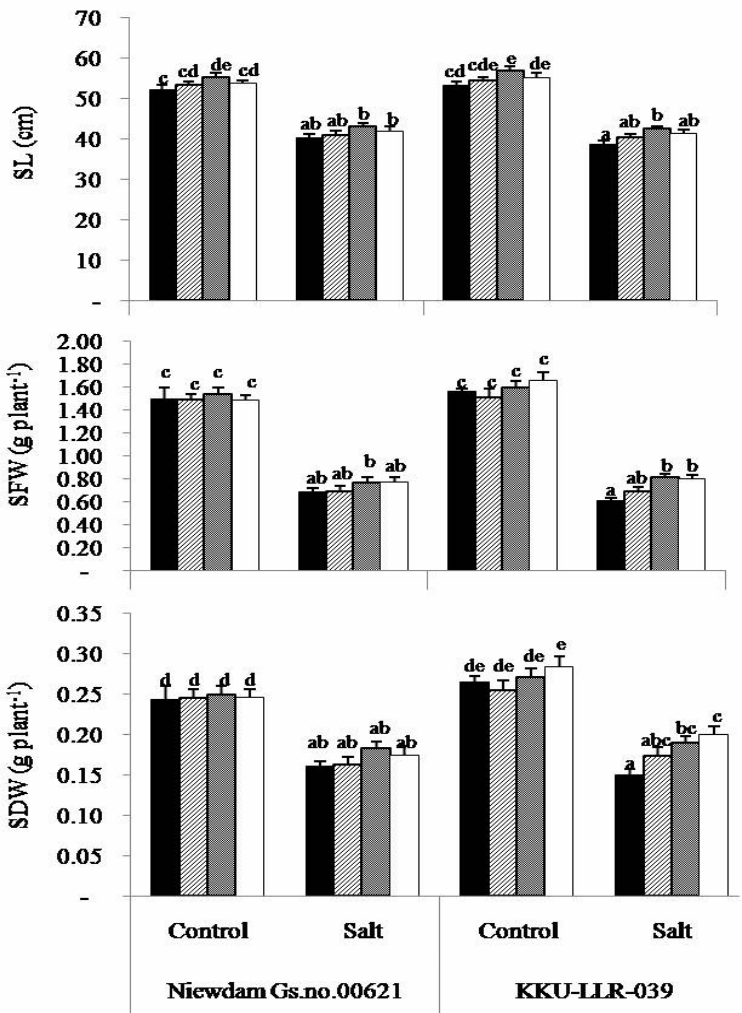

- Unprimed

Hydropriming salinity conditions. Moreover, Saleethong et al. (2011) observed that exogenously applied Spd contributed to the improvement of $\mathrm{K}^{+} / \mathrm{Na}^{+}$homeostasis in rice seedlings exposed to salt stress. Even though the mechanisms underlying the alleviation of the inhibitory effect of salinity by PAs have not yet been fully understood, several previous studies have suggested that exogenous application of PAs contributed to the reduced accumulation of toxic $\mathrm{Na}^{+}$in rice (Chattopadhayay et al., 2002; Quinet et al., 2010).

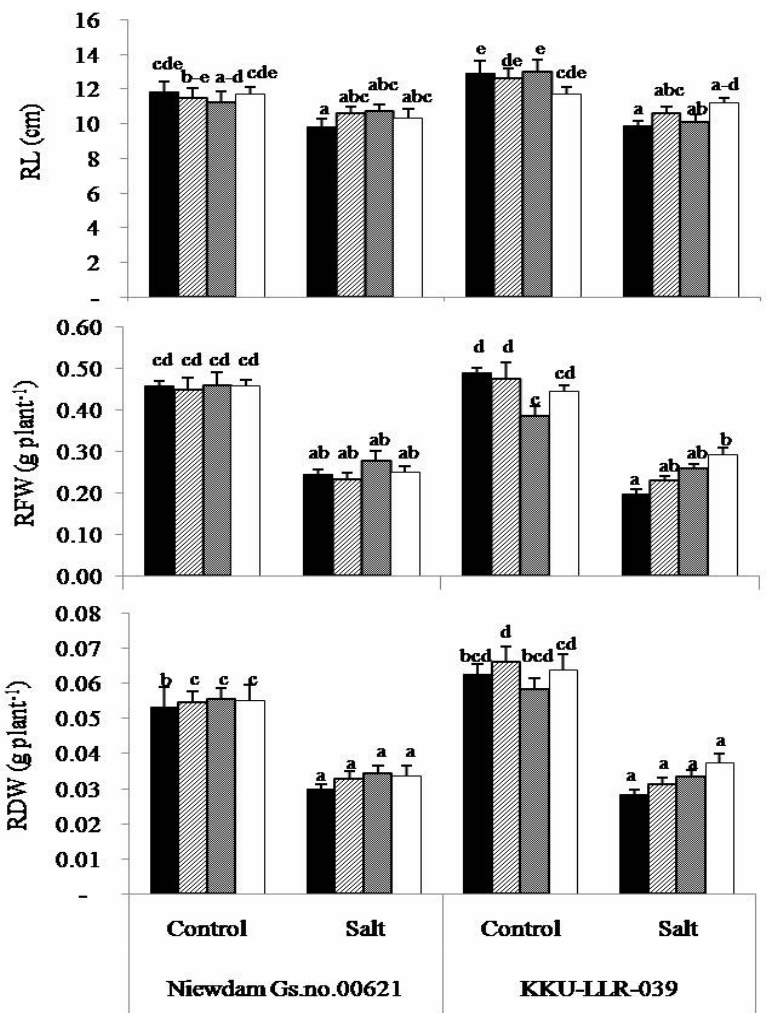

$\square$ Spd $\square \mathbf{G A}_{3}$

Fig. 1. Growth parameters of rice seedlings in saline conditions as affected by priming seeds with $\mathrm{H}_{2} \mathrm{O}$, Spd and $\mathrm{GA}_{3}$. Results are expressed as means \pm standard errors (SE). Bars with different letters are significantly different according to Duncan's multiple range tests $(\mathrm{p}<0.05)$.

Tab. 1. Effects of seed priming with $\mathrm{H}_{2} \mathrm{O}$, Spd and $\mathrm{GA}_{3}$ on some physiological traits of rice seedlings grown under salt stress $(\mathrm{NaCl} 150 \mathrm{mM})$ and non-stress conditions (Control)

\begin{tabular}{|c|c|c|c|c|c|c|c|c|c|c|}
\hline \multirow{2}{*}{ Cultivars } & \multirow{2}{*}{ Treatment } & \multicolumn{3}{|c|}{$\mathrm{Na}^{+} / \mathrm{K}^{+}$} & \multicolumn{3}{|c|}{ Proline (ugg $\left.{ }^{-1} \mathrm{FW}\right)$} & \multicolumn{3}{|c|}{ TAC $\left(\mathrm{mgg}^{-1} \mathrm{FW}\right)$} \\
\hline & & Control & Salt & $\%(+)$ & Control & Salt & $\%(+)$ & Control & Salt & $\%(-)$ \\
\hline \multirow{4}{*}{$\begin{array}{l}\text { Niewdam } \\
\text { Gs.no. } 00621\end{array}$} & Unprimed & $0.48^{\mathrm{a}}$ & $1.19^{\mathrm{d}}$ & 146.65 & $183^{a}$ & $1,777^{b}$ & 873.70 & $2.15^{f}$ & $0.95^{\mathrm{ab}}$ & 55.74 \\
\hline & Hydropriming & $0.47^{\mathrm{a}}$ & $1.14^{c}$ & 140.65 & $170^{2}$ & $1,696^{b}$ & 897.65 & $2.28^{f}$ & $1.13^{\mathrm{b}}$ & 50.26 \\
\hline & Spd $1 \mathrm{mM}$ & $0.45^{a}$ & $1.01^{b}$ & 126.79 & $181^{a}$ & $1,511^{\mathrm{b}}$ & 737.12 & $2.39^{f}$ & $1.22^{\mathrm{b}}$ & 48.96 \\
\hline & $\mathrm{GA}_{3} 0.43 \mathrm{mM}$ & $0.45^{a}$ & $1.03^{\mathrm{b}}$ & 128.60 & $187^{a}$ & $1,555^{b}$ & 731.55 & $2.32^{f}$ & $1.18^{\mathrm{b}}$ & 49.07 \\
\hline \multirow{4}{*}{ KKU-LLR-039 } & Unprimed & $0.49^{2}$ & $1.42^{f}$ & 188.98 & $194^{a}$ & $2,041^{\mathrm{b}}$ & 951.80 & $1.58^{c}$ & $0.64^{a}$ & 59.55 \\
\hline & Hydropriming & $0.46^{2}$ & $1.35^{f}$ & 193.91 & $184^{\mathrm{a}}$ & $1,968^{b}$ & 972.48 & $1.65^{\mathrm{dd}}$ & $0.76^{a}$ & 53.93 \\
\hline & Spd $1 \mathrm{mM}$ & $0.45^{a}$ & $1.19^{d \mathrm{~d}}$ & 164.40 & $186^{a}$ & $1,682^{b}$ & 804.30 & $1.88^{\text {de }}$ & $0.90^{\mathrm{b}}$ & 52.02 \\
\hline & $\mathrm{GA}_{3} 0.43 \mathrm{mM}$ & $0.46^{a}$ & $1.26^{\mathrm{de}}$ & 176.86 & $190^{\mathrm{a}}$ & $1,723^{b}$ & 809.23 & $1.90^{\mathrm{de}}$ & $0.91^{\mathrm{ab}}$ & 51.99 \\
\hline \multirow{2}{*}{ Cultivars } & \multirow{2}{*}{ Treatment } & \multicolumn{3}{|c|}{ Total-chl $\left(\mathrm{mgg}^{1} \mathrm{FW}\right)$} & \multicolumn{3}{|c|}{$\mathrm{Chl}-a\left(\mathrm{mgg}^{1} \mathrm{FW}\right)$} & \multicolumn{3}{|c|}{$\mathrm{Chl}-b\left(\mathrm{mgg}^{1} \mathrm{FW}\right)$} \\
\hline & & Control & Salt & $\%(-)$ & Control & Salt & $\%(-)$ & Control & Salt & $\%(-)$ \\
\hline \multirow{4}{*}{$\begin{array}{l}\text { Niewdam } \\
\text { Gs.no. } 00621\end{array}$} & Unprimed & $4.21^{\text {bed }}$ & $2.92^{\mathrm{a}}$ & 30.69 & $3.07^{\mathrm{cf}}$ & $1.98^{\mathrm{a}}$ & 35.71 & $1.12^{\text {atc }}$ & $0.93^{\text {ab }}$ & 16.83 \\
\hline & Hydropriming & $4.50^{\mathrm{dd}}$ & $3.41^{\text {bb }}$ & 24.19 & $3.25^{d f}$ & $2.34^{\mathrm{b}}$ & 28.18 & $1.23^{b c}$ & $1.06^{\text {bc }}$ & 13.60 \\
\hline & Spd $1 \mathrm{mM}$ & $4.57^{\mathrm{dd}}$ & $3.53^{\mathrm{ab}}$ & 22.78 & $3.34^{\mathrm{df}}$ & $2.41^{d x}$ & 27.79 & $1.21^{\mathrm{bc}}$ & $1.11^{\mathrm{d} x}$ & 8.90 \\
\hline & $\mathrm{GA}_{3} 0.43 \mathrm{mM}$ & $4.63^{\mathrm{dd}}$ & $3.60^{\mathrm{bb}}$ & 22.41 & $3.38^{\mathrm{dff}}$ & $2.43^{3 \mathrm{dx}}$ & 28.28 & $1.23^{\mathrm{bc}}$ & $1.16^{\mathrm{dic}}$ & 6.25 \\
\hline \multirow{4}{*}{ KKU-LLR-039 } & Unprimed & $4.82^{\mathrm{d}}$ & $3.63^{\mathrm{ab}}$ & 24.55 & $3.59^{f}$ & $2.68^{\text {lod }}$ & 25.49 & $1.21^{\mathrm{bc}}$ & $0.95^{\mathrm{ab}}$ & 21.71 \\
\hline & Hydropriming & $4.83^{\text {dd }}$ & $3.66^{\mathrm{b}}$ & 24.21 & $3.60^{f}$ & $2.78^{\text {bad }}$ & 22.56 & $1.21^{\mathrm{bc}}$ & $0.86^{\mathrm{a}}$ & 29.11 \\
\hline & Spd $1 \mathrm{mM}$ & $5.04^{\mathrm{d}}$ & $4.00^{b x}$ & 20.56 & $3.72^{\mathrm{f}}$ & $2.79^{\text {lod }}$ & 24.81 & $1.30^{c}$ & $1.19^{b c}$ & 8.41 \\
\hline & $\mathrm{GA}_{3} 0.43 \mathrm{mM}$ & $5.03^{\mathrm{d}}$ & $4.03^{b x}$ & 19.87 & $3.67^{f}$ & $2.92^{\text {be }}$ & 20.39 & $1.35^{c}$ & $1.10^{d x}$ & 18.43 \\
\hline
\end{tabular}

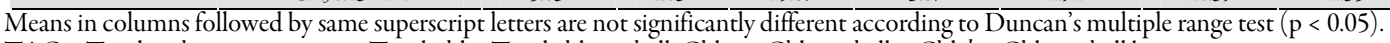

TAC $=$ Total anthocyanin content, Total-chl $=$ Total chlorophyll, Chl- $a=$ Chlorophyll $\mathrm{a}, \mathrm{Chl}-b=$ Chlorophyll b 
409

Tab. 2. Effects of seed priming with $\mathrm{H}_{2} \mathrm{O}$, Spd and $\mathrm{GA}_{3}$ on $\mathrm{H}_{2} \mathrm{O}_{2}$, TPC and antioxidant capacity (DPPH, ABTS and FRAP assays) of rice seedlings grown under salt stress $(\mathrm{NaCl} 150 \mathrm{mM})$ and non-stress conditions (Control)

\begin{tabular}{|c|c|c|c|c|c|c|c|c|c|c|c|c|c|c|c|c|}
\hline \multirow{2}{*}{ Cultivars } & \multirow{2}{*}{ Treatment } & \multicolumn{3}{|c|}{$\mathrm{H}_{2} \mathrm{O}_{2}$} & \multicolumn{3}{|c|}{$\mathrm{TPC}\left(\mathrm{mgg}^{-1} \mathrm{FW}\right)$} & \multicolumn{3}{|c|}{$\mathrm{DPPH}$ (\% radical scavenging) } & \multicolumn{3}{|c|}{ ABTS (\% radical scavenging) } & \multicolumn{3}{|c|}{$\mathrm{FRAP}\left(\mathrm{mmolTEg} \mathrm{g}^{-1} 100 \mathrm{FW}\right)$} \\
\hline & & Control & Salt & $\%(+)$ & Control & Salt & $\%(-)$ & Control & Salt & $\%(-)$ & Control & Salt & $\%(-)$ & Control & Salt & $\%(-)$ \\
\hline \multirow{4}{*}{$\begin{array}{l}\text { Niewdam } \\
\text { Gs. no. } \\
00621\end{array}$} & Unprimed & $5.26^{a}$ & $25.50^{b}$ & 384.47 & $1.16^{f}$ & $0.84^{\text {b }}$ & 27.75 & 81.09 & $67.51^{\mathrm{d}}$ & 16.75 & $62.16^{\mathrm{de}}$ & $45.54^{\mathrm{bc}}$ & 26.74 & $0.42^{\mathrm{gii}}$ & $0.29^{\mathrm{d}}$ & 30.22 \\
\hline & Hydropriming & $5.29^{2}$ & $24.35^{b}$ & 360.45 & $1.20^{\text {f }}$ & $0.86^{\text {lax }}$ & 28.20 & $81.64^{6}$ & $69.42^{\mathrm{d}}$ & 14.97 & $64.19^{\circ}$ & $48.18^{\mathrm{bx}}$ & 24.94 & $0.44^{\mathrm{hi}}$ & $0.30^{\mathrm{dd}}$ & 30.11 \\
\hline & Spd $1 \mathrm{mM}$ & $5.07^{\mathrm{a}}$ & $21.90^{b}$ & 331.75 & $1.35^{8}$ & $1.02^{2 \mathrm{de}}$ & 24.87 & $88.38^{8}$ & $76.58^{\text {f }}$ & 13.35 & $68.51^{e}$ & $53.14^{d \mathrm{~d}}$ & 22.44 & $0.48^{\mathrm{i}}$ & $0.35^{d f}$ & 27.14 \\
\hline & $\mathrm{GA}_{3} 0.43 \mathrm{mM}$ & $5.36^{2}$ & $22.72^{\mathrm{b}}$ & 323.77 & $1.34^{8}$ & $0.99^{\text {be }}$ & 25.94 & $88.15^{\mathrm{g}}$ & $74.75^{\mathrm{df}}$ & 15.21 & $68.15^{\mathrm{e}}$ & $52.75^{\mathrm{d}}$ & 22.61 & $0.48^{\mathrm{i}}$ & $0.34^{\text {df }}$ & 27.46 \\
\hline \multirow{4}{*}{$\begin{array}{l}\text { KKU- } \\
\text { LLR-039 }\end{array}$} & Unprimed & $6.44^{2}$ & $26.01^{b}$ & 303.73 & $0.95^{\text {bad }}$ & $0.71^{a}$ & 25.75 & $56.39^{\circ}$ & $29.68^{a}$ & 47.37 & $48.40^{b c}$ & $33.59^{a}$ & 30.58 & $0.31^{\mathrm{de}}$ & $0.13^{\mathrm{a}}$ & 58.51 \\
\hline & Hydropriming & $6.63^{a}$ & $24.76^{b}$ & 273.19 & $0.94^{\text {df }}$ & $0.74^{a}$ & 21.29 & $56.74^{c}$ & $38.43^{\mathrm{b}}$ & 32.27 & $46.83^{b c}$ & $38.62^{\mathrm{b}}$ & 17.52 & $0.31^{d d}$ & $0.18^{\mathrm{a}}$ & 41.55 \\
\hline & Spd $1 \mathrm{mM}$ & $5.99^{2}$ & $20.91^{\mathrm{b}}$ & 249.40 & $1.06^{\mathrm{df}}$ & $0.86^{\mathrm{ak}}$ & 19.00 & $71.23^{\text {de }}$ & $49.52^{c}$ & 30.47 & $52.75^{\mathrm{dd}}$ & $43.72^{\mathrm{bc}}$ & 17.11 & $0.39^{\text {fot }}$ & $0.24^{b}$ & 38.32 \\
\hline & $\mathrm{GA}_{3} 0.43 \mathrm{mM}$ & $6.08^{a}$ & $22.31^{\mathrm{b}}$ & 266.80 & $1.04^{\text {dff }}$ & $0.87^{\mathrm{ikx}}$ & 16.77 & $70.85^{\text {de }}$ & $50.40^{\circ}$ & 28.86 & $51.89^{\circ}$ & $44.54^{\mathrm{bc}}$ & 14.16 & $0.37^{\text {df }}$ & $0.25^{\mathrm{bc}}$ & 33.13 \\
\hline
\end{tabular}

Means in columns followed by same superscript letters are not significantly different according to Duncan's multiple range test ( $\mathrm{p}<0.05)$.

$\mathrm{TPC}=$ Total phenolic content. DPPH = 2,2' -diphenyl-1-picrylhydrazyl, ABTS = 2,2'-azinobis (3-ethylbenzothiazoline 6-sulfonic acid), FRAP $=$ Ferric reducing antioxidant power

Tab. 3. Mean squares from analysis of variance for growth traits, physiological characteristics and antioxidant capacity in two cultivars of rice seedlings grown under salt stress from unprimed seeds or seeds primed with $\mathrm{H}_{2} \mathrm{O}$, Spd and $\mathrm{GA}_{3}$

\begin{tabular}{|c|c|c|c|c|c|c|c|c|c|c|c|}
\hline Source of variation & $\mathrm{df}$ & $\begin{array}{l}\mathrm{SL} \\
(\mathrm{cm})\end{array}$ & $\begin{array}{l}\mathrm{RL} \\
(\mathrm{cm})\end{array}$ & $\begin{array}{c}\text { SFW } \\
\text { ( } \mathrm{g}^{-1} \text { plant) }\end{array}$ & $\begin{array}{c}\text { RFW } \\
\text { (g-1plant) }\end{array}$ & $\begin{array}{c}\text { SDW } \\
\text { ( } \mathrm{g}^{-1} \text { plant) }\end{array}$ & $\begin{array}{c}\text { RDW } \\
\text { (g-1 plant) }\end{array}$ & $\mathrm{Na}^{+} / \mathrm{K}^{+}$ & $\begin{array}{c}\text { Total-chl } \\
\left(\mathrm{mgg}^{-1} \mathrm{FW}\right)\end{array}$ & $\begin{array}{c}\text { Chl- } a \\
\left(\mathrm{mgg}^{-1} \mathrm{FW}\right)\end{array}$ & $\begin{array}{c}\text { Chl- } b \\
\left(\mathrm{mgg}^{-1} \mathrm{FW}\right)\end{array}$ \\
\hline Cultivars (C) & 1 & $2.93 \mathrm{~ns}$ & $13.868^{*}$ & $0.0705 \mathrm{~ns}$ & $0.00233 \mathrm{~ns}$ & $0.01136^{*}$ & $0.00077^{*}$ & $0.08925^{*}$ & $3.3658^{*}$ & $3.1301^{*}$ & $0.0033 \mathrm{~ns}$ \\
\hline Priming $(\mathrm{P})$ & 3 & $93.06^{*}$ & $0.537 \mathrm{~ns}$ & $0.1151^{*}$ & $0.00256 \mathrm{~ns}$ & $0.00512^{*}$ & $0.00014 \mathrm{~ns}$ & $0.02376^{*}$ & $0.6280 \mathrm{~ns}$ & $0.2336 \mathrm{~ns}$ & $0.1032^{*}$ \\
\hline Salt $(S)$ & 1 & $8298.97^{*}$ & $133.00^{*}$ & $31.7327^{*}$ & $2.02126^{*}$ & $0.32880^{*}$ & $0.3236^{*}$ & $4.29978^{*}$ & $19.5687^{*}$ & $13.31^{*}$ & $0.5732^{*}$ \\
\hline $\mathrm{C} \times \mathrm{P}$ & 3 & $1.03 \mathrm{~ns}$ & $0.063 \mathrm{~ns}$ & $0.0280 \mathrm{~ns}$ & $0.00991 \mathrm{~ns}$ & $0.0016 \mathrm{~ns}$ & $0.00006 \mathrm{~ns}$ & $0.00069 \mathrm{~ns}$ & $0.0980 \mathrm{~ns}$ & $0.0497 \mathrm{~ns}$ & $0.0303 \mathrm{~ns}$ \\
\hline $\mathrm{P} \times \mathrm{S}$ & 3 & $0.38 \mathrm{~ns}$ & $5.204 \mathrm{~ns}$ & $0.0301 \mathrm{~ns}$ & $0.02467^{*}$ & $0.00163 \mathrm{~ns}$ & $0.00010 \mathrm{~ns}$ & $0.01121^{*}$ & $0.0394 \mathrm{~ns}$ & $0.199 \mathrm{~ns}$ & $0.1801 \mathrm{~ns}$ \\
\hline $\mathrm{C} \times \mathrm{S}$ & 1 & $51.56^{*}$ & $10.360^{*}$ & $0.0778 \mathrm{~ns}$ & $0.00001 \mathrm{~ns}$ & $0.00247 \mathrm{~ns}$ & $0.00080^{*}$ & $0.09138^{*}$ & $0.0013 \mathrm{~ns}$ & $0.0633 \mathrm{~ns}$ & $0.4673 \mathrm{~ns}$ \\
\hline $\mathrm{C} \times \mathrm{P} \times \mathrm{S}$ & 4 & $8.08 \mathrm{~ns}$ & $6.119^{*}$ & $0.0306 \mathrm{~ns}$ & $0.01611^{*}$ & $0.00130 \mathrm{~ns}$ & $0.00018 \mathrm{~ns}$ & $0.01801^{*}$ & $0.00493 \mathrm{~ns}$ & $0.02193 \mathrm{~ns}$ & $0.01731 \mathrm{~ns}$ \\
\hline $\mathrm{CV}(\%)$ & & 6.72 & 12.58 & 14.78 & 24.20 & 15.56 & 23.33 & 4.75 & 12.18 & 13.67 & 16.02 \\
\hline
\end{tabular}

\begin{tabular}{|c|c|c|c|c|c|c|c|c|}
\hline Source of variation & $\mathrm{df}$ & $\begin{array}{c}\text { TAC } \\
\left(\mathrm{mgg}^{-1} \mathrm{FW}\right)\end{array}$ & $\begin{array}{c}\text { Proline } \\
\left.\text { (ugg }{ }^{-1} \mathrm{FW}\right)\end{array}$ & $\mathrm{H}_{2} \mathrm{O}_{2}$ & $\begin{array}{c}\text { TPC } \\
\left(\mathrm{mgg}^{-1} \mathrm{FW}\right)\end{array}$ & $\begin{array}{c}\text { DPPH } \\
\text { (\% inhibition) }\end{array}$ & $\begin{array}{c}\text { ABTS } \\
\text { (\%inhibition) }\end{array}$ & $\begin{array}{c}\text { FRAP } \\
\left(\mathrm{mmol} \mathrm{TEg}^{-1} 100\right. \\
\text { FW) }\end{array}$ \\
\hline Cultivars $(\mathrm{C})$ & 1 & $2.9096^{*}$ & $205889^{*}$ & $3.38 \mathrm{~ns}$ & $0.6376^{*}$ & $10432.1^{*}$ & $2615.42^{*}$ & $0.2134^{*}$ \\
\hline Priming $(\mathrm{P})$ & 3 & $0.2475^{*}$ & $89613.4^{*}$ & $18.06 \mathrm{~ns}$ & $0.1096^{*}$ & $682.6^{*}$ & $202.57^{*}$ & $0.0223^{*}$ \\
\hline Salt $(S)$ & 1 & $17.88^{*}$ & $3.893 \mathrm{E}+07^{*}$ & $5064.58^{*}$ & $1.17208^{*}$ & $4773.4^{*}$ & $2641.02^{*}$ & $0.30523^{*}$ \\
\hline $\mathrm{C} \times \mathrm{P}$ & 3 & $0.170^{*}$ & $3822.90 \mathrm{~ns}$ & $0.88 \mathrm{~ns}$ & $0.0023 \mathrm{~ns}$ & $96.7^{*}$ & $1.08 \mathrm{~ns}$ & $0.0013 \mathrm{~ns}$ \\
\hline $\mathrm{P} \times \mathrm{S}$ & 3 & $0.0027 \mathrm{~ns}$ & $91071.9^{*}$ & $13.03 \mathrm{~ns}$ & $0.00034 \mathrm{~ns}$ & 16.9ns & $15.10 \mathrm{~ns}$ & $0.00068 \mathrm{~ns}$ \\
\hline $\mathrm{C} \times \mathrm{S}$ & 1 & $0.1843^{*}$ & $1777031^{*}$ & $0.88 \mathrm{~ns}$ & $0.06850^{*}$ & $327.0^{*}$ & $144.45 \mathrm{~ns}$ & $0.00106 \mathrm{~ns}$ \\
\hline $\mathrm{C} \times \mathrm{P} \times \mathrm{S}$ & 4 & $0.04812 \mathrm{~ns}$ & $46277^{*}$ & $1.554 \mathrm{~ns}$ & $0.0183 \operatorname{lns}$ & $89.3^{*}$ & $42.39 \mathrm{~ns}$ & $0.00089 \mathrm{~ns}$ \\
\hline $\mathrm{CV}(\%)$ & & 13.32 & 9.16 & 24.25 & 10.10 & 7.96 & 11.90 & 11.57 \\
\hline
\end{tabular}

SL = Shoot length, RL = Root length, SFW = Shoot fresh weight, RFW = Root fresh weight, SDW = Shoot dry weight, RDW = Root dry weight, Total-chl = Tota chlorophyll content, Chl- $a=$ Chlorophyll a, Chl- $b=$ Chlorophyll b, TAC = Total anthocyanin content, TPC = Total phenolic content, DPPH $=2,2^{\prime}-$ diphenyl-1picrylhydrazyl, $\mathrm{ABTS}=2,2^{\prime}$-azinobis (3-ethylbenzothiazoline 6-sulfonic acid), FRAP = Ferric reducing antioxidant power.

Tab. 4. Pearson's correlation coefficient of growth traits, physiological characteristics and antioxidant capacity in two rice cultivars under saline conditions

\begin{tabular}{|c|c|c|c|c|c|c|c|c|c|c|c|c|c|c|c|c|c|}
\hline $\begin{array}{l}\text { Physiological } \\
\text { traits }\end{array}$ & SL & $\mathrm{RL}$ & SFW & SDW & RFW & RDW & $\mathrm{Na}^{+} / \mathrm{K}^{+}$ & Total-chl & $\mathrm{Chl}-a$ & Chl- $b$ & TAC & Proline & $\mathrm{H}_{2} \mathrm{O}_{2}$ & TPC & DPPH & ABTS & FRAP \\
\hline SL & 1 & .440 & $.893^{\prime \prime}$ & $.790^{\circ}$ & $.723^{\circ}$ & $.724^{\circ}$ & $-.802^{\prime \prime}$ & .290 & .028 & $.756^{\circ}$ & $.734^{\circ}$ & $-.885^{\prime \prime}$ & $-.911^{* \prime}$ & $.847^{*}$ & $.630^{\circ}$ & $.785^{\circ}$ & $.732^{\circ}$ \\
\hline $\mathrm{RL}$ & & 1 & .506 & $.669^{\circ}$ & $.700^{\circ}$ & $.817^{\prime \prime}$ & -.205 & .489 & .450 & .259 & .331 & -.344 & -.461 & .342 & .164 & .336 & .264 \\
\hline SFW & & & 1 & $.878^{*}$ & $.917^{\circ}$ & $.851^{*}$ & -.585 & .521 & .276 & .786 & .515 & $-.753^{\circ}$ & $-.955^{\circ \prime}$ & $.684^{\circ}$ & .414 & .610 & .550 \\
\hline SDW & & & & 1 & $.894^{*}$ & $.885^{\circ}$ & -.546 & .334 & .146 & .585 & .530 & $-.715^{\circ}$ & $-.780^{\circ}$ & $.689^{\circ}$ & .474 & $.625^{\circ}$ & .596 \\
\hline RFW & & & & & 1 & $.875^{\prime \prime}$ & -.292 & $.688^{\circ}$ & .537 & .603 & .261 & -.511 & $-.860^{\circ}$ & .459 & .136 & .361 & .291 \\
\hline RDW & & & & & & 1 & -.537 & .503 & .297 & $.690^{\circ}$ & .582 & $-.711^{\circ}$ & $-.779^{\circ}$ & $.667^{\circ}$ & .449 & .636 & .573 \\
\hline $\mathrm{Na}^{+} / \mathrm{K}^{+}$ & & & & & & & 1 & .197 & .479 & $-.661^{\circ}$ & $-.974^{\prime \prime}$ & $.964^{\circ}$ & .574 & $-.960^{\circ}$ & $-.951^{*}$ & $-.982^{\prime \prime}$ & $-.973^{\prime \prime}$ \\
\hline Total-chl & & & & & & & & 1 & $.942 "$ & .479 & -.221 & -.019 & $-.622^{\circ}$ & -.019 & -.435 & -.183 & -.296 \\
\hline Chl- $a$ & & & & & & & & & 1 & .155 & -.482 & .288 & -.362 & -.303 & $-.674^{\circ}$ & -.451 & -.555 \\
\hline Chl- $b$ & & & & & & & & & & 1 & .600 & $-.796^{\prime \prime}$ & $-.864^{\prime \prime}$ & $.730^{\circ}$ & .473 & $.628^{\circ}$ & .567 \\
\hline TAC & & & & & & & & & & & 1 & $-.933^{\prime \prime}$ & -.493 & $.927^{* \prime}$ & $.964^{\circ}$ & $.985^{\circ}$ & $.976^{*}$ \\
\hline Proline & & & & & & & & & & & & 1 & $.741^{\circ}$ & $-.977^{*}$ & $-.880^{*}$ & $-.962^{\prime \prime}$ & $-.938^{*}$ \\
\hline $\mathrm{H}_{2} \mathrm{O}_{2}$ & & & & & & & & & & & & & 1 & $-.679^{\circ}$ & -.346 & -.560 & -.482 \\
\hline TPC & & & & & & & & & & & & & & 1 & $.876^{\circ}$ & $.960^{\circ}$ & $.932^{* \prime}$ \\
\hline DPPH & & & & & & & & & & & & & & & 1 & $.961^{*}$ & $.986^{\circ}$ \\
\hline ABTS & & & & & & & & & & & & & & & & 1 & $.991^{*}$ \\
\hline
\end{tabular}




\section{Spd and $G A_{3}$ primingmodulatespigment accumulation}

The imposition of salinity caused a significant reduction in chlorophyll accumulation in both cultivars (Tab. 1). Salt stress generally causes the inhibition of pigment accumulation in plants owing to the instability of protein complex and destruction of chlorophyll by inducing the activity of chlorophyllase, chlorophyll-degrading enzyme (Reddy and Vora, 1986). Protective roles of Spd on reduction of chlorophyll loss was previously reported by Roychoudhury $e t$ al. (2011) and Saleethong et al. (2011). Salt stress also had a negative effect on TAC content. In seedlings grown from unprimed seeds, TAC contents in salt-stressed seedlings were reduced by $55.74 \%$ in 'Niewdam Gs. no. 00621' and $59.55 \%$ in 'KKU-LLR-039'. The findings in our study were in good agreement with an earlier study (Kachout et al., 2013) demonstrating that high salt concentrations $(180-260 \mathrm{mM})$ resulted in a significant reduction in anthocyanin contents in the leaves of Atriplex hortensis. Similarly, Trivellini et al. (2014) demonstrated that salt stress adversely affected the content of anthocyanins in Hibiscus rosasinensis L. (cv. Porto), resulting in a visually perceptible loss of color. By contrast, some previous studies have reported the contribution of salt stress to an increase in anthocyanin contents in plants (Eryilmaz, 2006; Daiponmak et al., 2010). In general, changes in anthocyanin contents in plants depend largely on the level of salinity plants are exposed to, plant species and various environmental conditions (Chalker-Scott, 1999). The adverse effects of salt stress on TAC contents were slightly alleviated in seedlings raised from all three priming treatments. Our data demonstrated that seedlings of both rice cultivars exposed to salinity showed decreased pigment accumulation, but those raised from seeds pre-treated with Spd and $\mathrm{GA}_{3}$ displayed improved accumulation of Chl- $a$, Chl- $b$ and total-Chl, as well as TAC under saline conditions.

Analysis of variance (Tab.3) revealed that the cultivar main effect was significant for TAC, total-Chl and Chl- $a$, but was not significant for $\mathrm{Chl}-b$. The interaction between cultivars and priming treatments $(\mathrm{C} \times \mathrm{P})$ was not significant for chlorophyll contents but was significant for TAC. For both cultivars, all three priming treatments had positive effects on maintaining the chlorophyll contents under salt stress (except the effects of hydropriming on Chl- $b$ content of 'KKU-LLR$\left.039^{\prime}\right)$. However, priming with $\mathrm{Spd}$ and $\mathrm{GA}_{3}$ were more effective than hydropriming. There was positive correlation of pigment accumulation with some growth traits (SL, SFW and RDW) under saline condition (Tab. 4). These findings in our study were well-supported by earlier reports (Ali $e t$ al., 2012; Ratnakar and Rai, 2014) that exogenous application of $\mathrm{GA}_{3}$ assisted in the restoration of altered pigment concentrations in saline conditions. Moreover, Saleethong et al. (2011) reported that improved accumulation of total-Chl exerted by exogenous Spd was observed in rice seedlings under saline conditions. Roychoudhury et al. (2011) disclosed that salt-induced accumulation of anthocyanins in seedlings of three rice cultivars was substantially enhanced by exogenously applied Spd.

\section{Spd and $G A_{3}$ priming modulates proline content}

Proline accumulation is a well-known mechanism numerous plants have evolved to counteract salt stress. In addition to its crucial roles in protecting the subcellular structures and mediating osmotic adjustment in stressed conditions (Parvaiz and Satyawati, 2008; Rao et al., 2013a), proline is known to play diverse adaptive roles including protection of cellular functions by scavenging ROS (Smirnoff and Cumbes, 1989). Our data showed that seedlings under saline conditions accumulated higher levels of proline when compared with those under non-saline conditions. Salinity caused a dramatic increase (873\% in 'Niewdam Gs. no. 00621' and $951 \%$ in 'KKU-LLR-039') in proline content in both cultivars (Tab. 1). However, seedlings raised from seeds presoaked in Spd and $\mathrm{GA}_{3}$ was noted to exhibit decreased accumulation of proline in both rice cultivars in saline conditions (Tab. 1). Strong positive correlations were noted between proline content and the two salt-damaged parameters i.e. $\mathrm{Na}^{+} / \mathrm{K}^{+}$and $\mathrm{H}_{2} \mathrm{O}_{2}$, while significant negative correlations were found between proline and seedling growth, TAC, TPC and antioxidant activity under salt conditions (Tab. 4). The cultivar main effect, salt and priming treatments were significant for proline. Nevertheless, there were no cultivars $\mathrm{x}$ priming treatments $(\mathrm{C} \times \mathrm{P})$ interactions for proline (Tab. 3). The findings in our study were consistent with an earlier study (Saleethong et al., 2011) that exogenous Spd tangibly decreased the level of proline in rice seedlings under saline conditions. Moreover, exogenous application Spd was found to reduce the level of salt-induced proline, associated with improved leaf yield, chlorophyll and photosynthetic efficiency, in mulberry (Das et al., 2002). However, Duan et al. (2008) reported that exogenously applied Spd substantially increased the level of proline in cucumber roots in saline conditions.

\section{Spd and $\mathrm{GA}_{3}$ priming alters $\mathrm{H}_{2} \mathrm{O}_{2}$}

In general, the imposition of salinity causes damage to plant tissues as a result of excessive ROS like $\mathrm{H}_{2} \mathrm{O}_{2}$ produced at a high rate due to ion imbalance and hyperosmotic stresses. ROS accumulation leads to lipid oxidation and has a negative effect on cellular metabolism and physiology, thus detrimentally affecting the membrane integrity (Munns et al., 2006). The imposition of salinity significantly increased $\mathrm{H}_{2} \mathrm{O}_{2}$ content in both cultivars. Under the control (non-stressed conditions), $\mathrm{H}_{2} \mathrm{O}_{2}$ content tended to be higher in 'KKU-LLR-039' than 'Niewdam Gs. no. 00621'. On the other hand, the lessened production of $\mathrm{H}_{2} \mathrm{O}_{2}$ was observed in seedlings of both cultivars raised from seeds pre-soaked in Spd and $\mathrm{GA}_{3}$ (Tab. 2). Previous research has indicated that exogenously applied PAs resulted in decreased production of $\mathrm{H}_{2} \mathrm{O}_{2}$ under salinity (Verma and Mishra, 2005). Farooq et al. (2009) also found that under drought stress the curtailed production of $\mathrm{H}_{2} \mathrm{O}_{2}$ was observed in rice seedlings raised from seeds pre-treated with Spd. However, analysis of variance showed that the main effect of cultivars, priming treatments and their interactions were not significant for $\mathrm{H}_{2} \mathrm{O}_{2}$ (Tab. 3).

\section{Spd and $G A_{3}$ priming modulates phenolic compound accumulation and antioxidant capacity}

Phenolic compounds exhibit antioxidant capacity inactivating lipid free radicals or by preventing the decomposition of hydroperoxides into free radicals (Pokorny et al., 2001). In our study, salinity caused a marked reduction in TPC in both rice cultivars (Tab. 2). The findings in our study were consistent with one previous study (Rao et al., 2013b) that the accumulation of phenolic compounds in wheat plants was reduced under salt stress. Moreover, salinity stress was 
411

reported to cause an inhibitory effect on the production of bound phenolic compounds in wheat and bean cultivars (Radi et al., 2013). On the other hand, Valifard et al. (2014) elucidated that the accumulation of phenolic compounds was induced under moderate salinity whilst high salinity caused a reduction in phenolic contents, as observed for Salvia mirzayanii. Phenolic compounds may also be associated with antioxidant potential, due to their ability to directly protect the damage in plant cell (Wahid and Ghazanfar, 2006; Wahid, 2007). Seed priming with $\mathrm{H}_{2} \mathrm{O}$, Spd and $\mathrm{GA}_{3}$ tended to improve the amount of TPC in both cultivars (Tab. 2). The significantly improved accumulation of TPC was noted in 'Niewdam Gs. no. 00621' in Spd treatments when compared with the unprimed treatment, but significant improvement in the accumulation of TPC was not observed in $\mathrm{GA}_{3}$ treatments. The findings in our study corroborate one previous study (Farooq et al., 2009) that exogenous Spd substantially increased the production of phenolic compounds in rice under drought stress. The activation of several enzymatic and non-enzymatic antioxidants can detoxify ROS in stressed cells (Blokhina et al., 2003). In general, plant genotypes with stress-resistant traits possess superior ability of ROS scavenging through the production of higher levels of antioxidants. The positive roles of phenolic compounds on plant growth were evidenced by its strong positive correlations with shoot length, seedling fresh and dry weight, chl- $b$, TAC and its negative correlations with the negative physiological indicators for salt sensitivity, the $\mathrm{Na}^{+} / \mathrm{K}^{+}$and proline content.

The antioxidant activity observed for both cultivars was significantly reduced under saline conditions (Tab. 2). Pretreatment with Spd and $\mathrm{GA}_{3}$, however, contributed to improved antioxidant performance. Seedlings of the salttolerant cultivar ('Niewdam Gs. no. 0062') raised from seeds primed with Spd displayed the significantly enhanced activity of DPPH, whereas significant improvement in antioxidant activity was not observed in seedlings raised from seeds pretreated with $\mathrm{H}_{2} \mathrm{O}$ and $\mathrm{GA}_{3}$ in saline conditions. Seedlings of the salt-sensitive cultivar ('KKU-LLR-039') were raised from seeds pre-soaked in Spd and $\mathrm{GA}_{3}$ showed a significant increase in the activities of DPPH, ABTS and FRAP under both saline and non-saline conditions. The findings in our study were consistent with an earlier study that exogenously applied Spd contributed to the elevated activity of antioxidant enzymes in rice (Roychoudhury et al., 2011). Moreover, Choudhary et al. (2012) elucidated that exogenous application of Spd was noted to enhance the total antioxidant system in radish under $\mathrm{Cu}$ stress, as suggested by increased levels of DPPH and FRAP. Variations in antioxidant capacity were observed (Tab. 3). The main effect of cultivars and priming treatments were significant for TPC, DPPH, ABTS and FRAP. The interactions between cultivars and priming treatment were significant for only DPPH. Simultaneously phenolic compounds were positively correlated with the antioxidant capacity (DPPH, ABTS and FRAP) under saline condition (Tab. 4). Although the mechanisms underlying the functions of PAs in salt stress are not fully understood, recent studies have elucidated that exogenous application of PAs was observed to reduce saltinduced oxidative damage by activating antioxidant enzymes in Virginia pine (Tang and Newton, 2005). In addition, our results are supported by the findings reported by Verma and Mishra (2005) who found that PAs could enhance activities of several antioxidant enzymes and non-enzymatic antioxidants, leading to less stress damage in Brassica juncea seedlings.

Although the mechanisms of actions is still unclear, our results suggested, in accordance with earlier reports, that exogenous application of Spd and $\mathrm{GA}_{3}$ as seed priming is an effective approach for mitigating deleterious effects of salinity in rice seedlings. Seed priming with $\mathrm{GA}_{3}$ helps accelerate metabolic reactions before germination process by modulating ion uptake and partitioning, enhancing ABA catabolism and modulating hormone homeostasis, thus allowing enhanced seed germination and seedling growth under salinity stress conditions (Gonai et al., 2004; Iqbal and Ashraf, 2013). Exogenous Spd has been found to alleviate salt-stress damage by protecting membrane phospholipids, scavenging free radicals, activating antioxidant enzymes and prevented $\mathrm{NaCl}$-induced $\mathrm{K}^{+}$efflux by inhibition of non-selective cation channel (Shabala et al., 2007; Groppa and Benavides, 2008). Moreover, Li et al. (2014) revealed that beneficial effects of Spd as the seed priming agent were associated with its effects on improving starch metabolism by enhancing amylase activities which is the well-known effect of GAs. Although Spd and $\mathrm{GA}_{3}$ belong to different groups of hormones, their beneficial effects as seedpriming agents are strikingly similar. It would be extremely interesting to further elucidate the mechanisms of cross-talking between $\mathrm{GA}_{3}$ and Spd to increase our understanding of integrating hormonal actions on alleviation of damaging effects of salinity stress.

\section{Conclusion}

The imposition of salinity caused a significant reduction in growth parameters in both rice cultivars. Pre-treatment of seeds with Spd and $\mathrm{GA}_{3}$ improved growth of seedlings of both rice cultivars, with the salt-sensitive cultivars exhibiting apparent improvement. Salt stress was also observed to cause adverse physiological and biochemical changes in seedlings of both cultivars. Pre-soaking of seeds in Spd and $\mathrm{GA}_{3}$ contributed to improved ion homeostasis as well as delaying loss of pigment accumulation. Moreover, Spd and $\mathrm{GA}_{3}$ priming treatments reduced the production of $\mathrm{H}_{2} \mathrm{O}_{2}$, but enhanced the antioxidant systems in rice plants exposed to salt stress. Based on our results, Spd was more effective than $\mathrm{GA}_{3}$ in improving salt tolerance in seedlings of both rice cultivars. It was noted that the promotive effects of Spd and $\mathrm{GA}_{3}$ on seedling growth in this study were quantitatively small which may limit the practical application of these compounds. Nevertheless, the wide array of physiological parameters investigated, particularly the antioxidant capacities, provided a new set of useful data with respect to the beneficial actions of hormonal priming.

\section{Acknowledgments}

This research was supported by a grant from the Food and Functional Food Cluster under the National Research University Project of Thailand's Office of the Higher Education Commission and Salt-tolerant Rice Research Group, Khon Kaen University. The authors are grateful to Dr. Poramet Buntaung of the Faculty of Agriculture of Khon Kaen University for kindly providing the rice seed material used in this study. 


\section{References}

Abdel-Aal EM, Hucl PA (1999). Rapid method for quantifying total anthocyanins in blue aleurone and purple pericarp wheats. Cereal Chem 76:350-354.

Afzal I, Butt A, Rehman HU, Basra SMA, Afzal A (2012). Alleviation of salt stress in fine aromatic rice byseed priming. Aust J Crop Sci 6(10): 1401-1407.

Alcázar R, Altabella T, Marco F, Bortolotti C, Reymond M, Koncz C, Carrasco P, Tiburcio AF (2010). Polyamines: molecules with regulatory functions in plant abiotic stress tolerance. Planta 231:1237-1249.

Ali HM, Siddiqui MH, Basalah MO, Al-Whaibi MH, Sakran AM, Al-Amri A (2012). Effects of gibberellic acid on growth and photosynthetic pigments of Hibiscus sabdariffa L. under salt stress. Afr J Biotechnol 11:800-804.

Arnon DI (1949). Copper enzymes in isolated chloroplasts. Polyphenyloxidase in Beta vulgaris. Plant Physiol 24:1-15.

Bates IS, Waldern RP, Teare ID (1973). Rapid determination of free proline for water stress studies. Plant Soil 39:205-207.

Bradford KJ (1986). Manipulation of seed water relations via osmotic priming to improve germination under stress conditions. Hort Sci 21:1105-1111.

Brand-Williams W, Cuvelier ME, Berset C (1995). Use of free radical method to evaluate antioxidant activity. LWT - Food Sci Technol 28:25-30.

Benzie IFF, Strain JJ (1996). The ferric reducing ability of plasma (FRAP) as a measure of 'antioxidant power': The FRAP assay. Anal Biochem 239:70-76.

Blokhina O, Virolainen E, Fagerstedt KV (2003). Antioxidants, oxidative damage and oxygen deprivation stress: a review. Ann Bot 91:179-194.

Blumwald E (2000). Sodium transport and salt tolerance in plants. Curr Opin Cell Biol 12:431-434.

Bouchereau A, Aziz A, Larher F, Martin-Tanguy J (1999). Polyamines and environmental challenges: recent development. Plant Sci 140:103-125.

Chalker-Scott L (1999). Environmental significance of anthocyanins in plant stress responses. Photochem Photobiol 70(1):1-9.

Chattopadhayay MK, Tiwari BS, Chattopadhyay G, Bose A, Sengupta DN, Ghosh B (2002). Protective role of exogenous polyamines on salinity-stressed rice (Oryza sativa) plants. Physiol Plant 116:192-199.

Choudhary SP, Oral HV, Bhardwaj R, Yu JQ, Tran LS (2012). Interaction of brassinosteroids and polyamines enhances copper stress tolerance in raphanus sativus. J Exp Bot 63:5659-5675.

Daiponmak W, Theerakulpisut P, Thanonkao P, Vanavichit A, Prathepha P (2010). Changes of anthocyanin cyanidin-3glucoside content and antioxidant activity in Thai rice varieties under salinity stress. Sci Asia 36:286-291.

Das C, Sengupta T, Chattopadhyay S, Setua M, Das NK, Saratchandra B (2002). Involvement of kinetin and spermidine in controlling salinity stress in mulberry (Morus alba L. cv. S1). Acta
Physiol Plant 24:53-57.

Duan J, Li J, Guo S, Kang Y (2008). Exogenous spermidine affects polyamine metabolism in salinity-stressed Cucumis sativus roots and enhances short-term salinity tolerance. J Plant Physiol 165:1620-1635.

Eryilmaz F (2006). The relationships between salt stress and anthocyanin content in higher plants. Biotechnol Biotech Eq 20(1):47-52.

Farooq M, Basra SMA, Wahid A, Khaliq A, Kobayashi N (2010). Rice seed invigoration: a review, p. 137-175. In: Organic farming, pest control and remediation of soil pollutants. Springer, Netherlands.

Farooq M, Basra SMA, Hussain M, Rehman H, Saleem BA (2007). Incorporation of polyamines in the priming media enhances the germination and early seedling growth in hybrid sunflower (Helianthus annuus L.). Int J Agr Biol 9:868-872.

Farooq M, Wahid A, Lee D-J (2009). Exogenously applied polyamines increase drought tolerance of rice by improving leaf water status, photosynthesis and membrane properties. Acta Physiol Plant 31:937-945.

Gonai T, Kawahara S, Tougou M, Satoh S, Hashiba T, Hirai N, Kawaide H, Kamaiya Y, Yoshioka T (2004). Abscisic acid in the thermoinhibition of lettuce seed germination and enhancement of its catabolism by gibberellin. J Exp Bot 55:111-118.

Ghodrat V, Rousta MJ (2012). Effect of priming with gibberellic acid $\left(\mathrm{GA}_{3}\right)$ on germination and growth of Corn (Zea mays $\mathrm{L}$.) under saline conditions. Int J Agr Crop Sci 4(13):882-885.

Groppa MD, Benavides MP (2008). Polyamines and abiotic stress: recent advances. Amino Acid 34:35-45.

Iqbal M, Ashraf M (2013). Gibberellic acid mediated induction of salt tolerance in wheat plants: Growth, ionic partitioning, photosynthesis, yield and hormonal homeostasis. Environ Exp Bot 86:76-85.

Kachout SS, Hamza KJ, Karray N, Bouraoui NK, Leclerc JC, Ouerghi $Z$ (2013). Salt-induced changes in antioxidative enzyme activities in shoot tissues of two Atriplex varieties. Not Bot Horti Agrobo 41(1):115-121.

Khajeh-Hosseini M, Powell AA, Bimgham IJ (2003). The interaction between salinity stress and seed vigor during germination of soybean seeds. Seed Sci Technol 31:715-725.

Khan HA, Ziaf K, Amjad M, Iqbal Q (2012). Exogenous application of polyamines improves germination and early seedling growth of hot pepper. Chil J Agr Res 72:429-433.

Koornneef M, Bentsink L, Hilhorst H (2002). Seed dormancy and germination. Curr Opin Plant Biol 5:33-36.

Kusano T, Berberich T, Tateda C, Takahashi Y (2008). Polyamines: essential factors for growth and survival. Planta 228:367-381.

Li Z, Peng Y, Zhang X-Q, Ma X, Huang L-K, Yan Y-H (2014). Exogenous spermidine improves seed germination of white clover under water stress via involvement in starch metabolism, antioxidant defenses and relevant gene expression. Molecules 19:18003-18024.

Magome H, Yamaguchi S, Hanada A, Kamiya Y, Oda K (2004). 
413

Dwarf and delayed-flowering 1, a novel Arabidopsis mutant deficient in gibberellin biosynthesis because of overexpression of a putative AP2 transcription factor. Plant J 37:720-729.

Munns R, James RA, Lauchli A (2006). Approaches to increasing the salt tolerance of wheat and other cereals. J Exp Bot 57:1025-1043.

Parvaiz A, Satyawati S (2008). Salt stress and phyto-biochemical responses of plants - a review. Plant Soil Environ 54:89-99.

Pedranzani H, Racagni G, Alemano S, Miersch O, Ramírez I, PeñaCortés H, Taleisnik E, Machado-Domenech E, Abdala G (2003). Salt tolerant tomato plants show increased levels of jasmonic acid. Plant Growth Regul 41:149-158.

Pokorny J, Yanishlieva N, Gordon M (2001). Antioxidants in food, practical applications. Woodhead Publishing Limited, Cambridge, 1-3 p.

Quinet M, Ndayiragije A, Lefevre I, Lambillotte B, Dupont-Gillain CC, Lutts S (2010). Putrescine differently influences the effect of salt stress on polyamine metabolism and ethylene synthesis in rice cultivars differing in salt resistance.J Exp Bot 61:2719-2733.

Rao PS, Mishra B, Gupta SR (2013a). Effect of salinity and alkalinity on grain quality of tolerant, semi-tolerant and sensitive rice genotypes. Rice Sci 20:284-291.

Rao A, Ahmad SD, Sabir SM, Shahid Awan S, Shah AH, Khan MF, Khan SA, Shafique S, Arif S, Abbas SR, Gohar M (2013b). Antioxidant activity and lipid peroxidation of selected wheat cultivars under salt stress. J Med Plants Res 7(4):155-164.

Radhakrishnan R, Lee IJ (2014). Effect of low dose of spermidine on physiological changes in salt-stressed cucumber plants. Russian J Plant Physiol 61:90-96.

Radi AA, Farghaly FA, Hamada AM (2013). Physiological and biochemical responses of salt-tolerant and salt-sensitive wheat and bean cultivars to salinity. J Biol Earth Sci 3(1):72-73.

Ratnakar A, Rai A (2014). Improvement of salt tolerance in Trigonella foenum-graecum L. var. PEB by plant growth regulators. J Stress Physiol Biochem 10(2):135-143.

Razem FA, Baron K, Hill RD (2006). Turning on gibberellin and abscisic acid signaling. Curr Opin Plant Biol 9:454-459.

Re R, Pellegrini N, Proteggente A, Pannala A, Yang M, Rice-Evans C (1999). Antioxidant activity applying an improved ABTS radical cation decolourization assay. Free Radic Biol Med 26:1231-1237.

Reddy MP, Vora AB (1986). Changes in pigment composition. Hill reaction activity and saccharides metabolism in bajra (Penisetum typhoides $\mathrm{S} \& \mathrm{H}$ ) leaves under $\mathrm{NaCl}$ salinity. Photosynthetica 20:50-55.

Roychoudhury A, Basu S, Sengupta DN (2011). Amelioration of salinity stress by exogenously applied spermidine or spermine in three varieties of indica rice differing in their level of salt tolerance. J Plant Physiol 168:317-328.

Saleethong P, Sanitchon J, Kong-ngern K, Theerakulpisut P (2011). Pretreatment with spermidine reverses inhibitory effects of salt stress in two rice (Oryza sativa L.) cultivars differing in salinity tolerance. Asian J Plant Sci 10:245-254.
Shabala S, Cuin TA, Pottosin I (2007). Polyamines prevent $\mathrm{NaCl}$ induced $\mathrm{K}+$ efflux from pea mesophyll by blocking non-selective cation channels. FEBS Lett 581:1993-1999.

Singleton VL, Orthofer R, Lamuela-Raventos RM (1999). Analysis of total phenols and other oxidation substrates and antioxidants by means of Folin-Ciocalteu reagent. Meth Enzymol 299:152-178.

Smirnoff N, Cumbes QJ (1989). Hydroxyl radical scavenging activity of compatible solutes. Phytochemistry 28:1057-1060.

Sultana B, Anwar F, Ashraf M (2009). Effect of extraction solvent/technique on the antioxidant activity of selected medicinal plant extracts. Molecules 14:2167-2180.

Sun Q, Wang JH, Sun BQ (2007). Advances on seed vigor physiological and genetic mechanisms. Agric Sci China 6:10601066.

Tang W, Newton RJ (2005). Polyamines reduce salt-induced oxidative damage by increasing the activities of antioxidant enzymes and decreasing lipid peroxidation in Virginia pine. Plant Growth Regul 46:31-43.

Trivellini A, Gordillo B, Rodríguez-Pulido FJ, Borghesi E, Ferrante A, Vernieri P, Quijada-Morín N, González-Miret ML, Heredia FJ (2014). Effect of salt stress in the regulation of anthocyanins and color of Hibiscus flowers by digital image analysis. J Agric Food Chem 62(29):6966-6974.

Valifarda M, Mohsenzadeh S, Kholdebarin B, Rowshan V (2014). Effects of salt stress on volatile compounds, total phenolic content and antioxidant activities of Salvia mirzayanii.

S Afr J Bot 93:92-97.

Velikova V, Yordanov I, Edreva A (2000). Oxidative stress and some antioxidant systems in acid rain-treated bean plants: protective role of exogenous polyamines. Plant Sci 151:59-66.

Verma S, Mishra SN (2005). Putrescine alleviation of growth in salt stressed Brassica juncea by inducing antioxidative defense system. J Plant Physiol 162:669-677.

Wahid A (2007). Physiological implications of metabolites biosynthesis in net assimilation and heat stress tolerance of sugarcane (Saccharum officinarum) sprouts. J Plant Res 120:219228.

Wahid A, Ghazanfar A (2006). Possible involvement of some secondary metabolites in salt tolerance of sugarcane. J Plant Physiol 163:723-730.

Wen F-P, Zhang Z-H, Bai T, Xu Q, Pan Y-H (2010). Proteomics reveals the effects of gibberellic acid $\left(\mathrm{GA}_{3}\right)$ on salt-stressed rice (Oryza sativa L.) shoots. Plant Sci 178:170-175.

Zhu H, Ding GH, Fang K, Zhao FG, Qin P (2006). New perspective on the mechanism of alleviating salt stress byspermidine in barleyseedlings. Plant Growth Regul 49:147-156. 